\title{
Some variation of the twisted cord illusion and their analyses**
}

\author{
THADDEUS M. COWAN $\dagger$ \\ Kansas State University, Manhattan, Kansas 66506
}

\begin{abstract}
Two approaches to the analysis of the twisted cord illusion are discussed. One suggests that the illusion is produced by embedded illusions (e.g., Zollner's illusion); the other points to nonillusion features such as the lines of the cord strands. A number of novel variations of the illusion are presented, and four experiments using these variations are described in an attempt to discern which of the two approaches was more viable. The implications of these results for various theories. including a multicomponent theory, are discussed.
\end{abstract}

Ever since Fraser discovered the twisted cord illusion in 1908 , it has been regarded as something of a curiosity. Few have attempted any systematic study of the illusion since Fraser; it has been kept alive mainly by its inclusion in introductory texts as an enticement for the curiosities of students. What may have been a near rediscovery by Dufour (1930) and a paper by Hyde (1929) on Munsterberg's checkerboard illusion, a related phenomenon, are exceptions, but these were more descriptive than analytical. More recently, Imai (1962) and Adam (1964) have reported effects which are essentially rediscoveries of Fraser's linear forms of his illusion. Also some interesting variations of the twisted cord illusion have been constructed by Parola and some of his students (1969).

A form of the twisted cord illusion, one that closely resembles Fraser's original figure, is shown in Fig. 1a. The twisted cord looks spiraled to most of those observing it, but in fact it forms a set of concentric rings. (One can easily demonstrate this for himself by placing a pencil tip on any ring and tracing it carefully. Eventually he will return to the starting point.) The number of different spiral components in the figure is enough to discourage even the most courageous, which is possibly why investigators have avoided this problem. Fraser, undaunted by these complexities, drew several conclusions regarding the illusion, some of which may eventually prove to be as insightful as they were bold. These will be introduced and discussed here, then referred to periodically throughout this paper.

At the close of his paper, Fraser drew the following conclusions regarding the illusion:

(a) There is no suggestion of perspective in the twisted cord figure which some writers have used to explain a variety of other illusions. This is a puzzling statement. Moving from outer to inner edge, the diminishing distances between the rings and the shrinking widths of the rings themselves provide strong distance cues. One

*I am indebted to the referees of this paper for a number of suggestions concerning related studies and possible approaches to the data. They should not be held responsible for errors of interpretation, however.

+ Requests for reprints should be sent to Thaddeus .I. Cowan Department of Psychology, Kansas State University. Manhattan. Kansas 66506. can easily imagine that he is looking down a tube in Fig. 1a. Yet Fraser's implication that distance cues cannot explain the illusion was substantially correct for reasons other than those he suggested. The presence of distance cues in Fig. 1a merely transforms the apparent spiral to an apparent helix. but in no way can the distance cues be used to explain the illusion. In the course of constructing a number of these figures, several were made in which the twisted cord rings were equally wide and placed apart by equal distances. The illusion was not destroyed or greatly weakened. One of these figures is shown in Fig. $2 .^{1}$

(b) In whatever way the illusion is related to Zollner's illusion, it is different in one important aspect, and that is, Zöllner's illusion is lost with steady fixation, whereas the twisted cord illusion is modified but never abolished. Fraser was referring to the lay of the "units of direction" across the circular lines of the twisted cord. These units of direction are the "strands" of the cord, and one with its points intact is shown in the inset in the lower right-hand corner of Fig. 1a. Zöllner's illusion, shown in Fig. 3a, produces an apparent deviation of lines away from the angle of the inducing lines. In Fraser's figure, however, the lines apparently bend in the direction of the inducing lines-an effect which, as Imai (1962) and Adam (1964) pointed out, is completely contrary to the Zöllner effect. Fraser, in his twisted cord article, demonstrated the illusory effects of the linear forms of these slanting lines by the LIFE figures reproduced in Fig. 3b. The illusion in Fig. $3 b$ will be spoken of in this paper as the reversed Zöllner illusion. Although Adam mentions the twisted cord illusion, she did not explicitly deal with the curved form of it (only a linear form related to those found in the LIFE figure). It is likely that she (and Imai) would have evoked the reversed Zöllner illusion to explain the twisted cord effect. The exact way in -which this illusion might produce the twisted cord effect will be described in detail later in this paper.

Getting back to Fraser's main point, the apparent lack of satiation is not necessarily linked to the fact that there may be a Zöllner illusion embedded in his figure. Furthermore, there may be satiation effects, but they require extensive fixation times. This possibility is based 

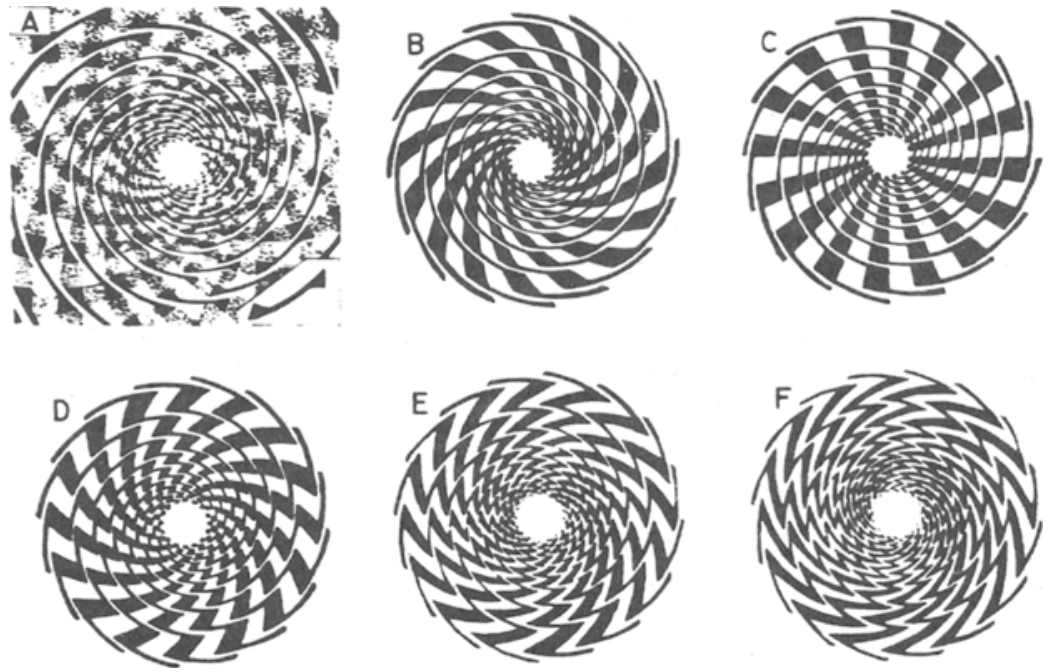

Fig. 1. Variations of the secondary spirals. (a) Fraser's original figure. (b) The stripe spirals turn through approximately $+90 \mathrm{deg}(+$ means in the direction of the illusion) and the stepped spirals turn through about +270 deg. (c) Stripe spirals: 0 deg. Stepped spirals: +180 deg. (d) Stripe spirals: -90 deg. Stepped spirals: $+90 \mathrm{deg}$. (e) Stripe spirals: $-180 \mathrm{deg}$. Stepped spirals: 0 deg. (f) Stripe spirals: -270 deg. Stepped spirals: $-90 \mathrm{deg}$. on my own phenomenological observations, however. After spending several hours constructing a number of clockwise forms of this illusion, I found that in some cases the illusion all but disappeared (Fig. $1 \mathrm{~b}$ and Fig. 2 in particular). Yet the counterclockwise versions of these were quite overwhelming-even to the point of being far stronger than my initial perceptions (as I remember them).

(c) Eye movements are eliminated as a factor, since the illusion persists at a 1/8-to 1/2-sec flash exposure. It is not clear whether Fraser took precautions to eliminate an afterimage, but given that he did, $125 \mathrm{msec}$ (a) even though it eliminates eye movements, would provide enough time for an efferent command to be readied (Festinger, Ono, Burnham, \& Bamber, 1967), and (b) is

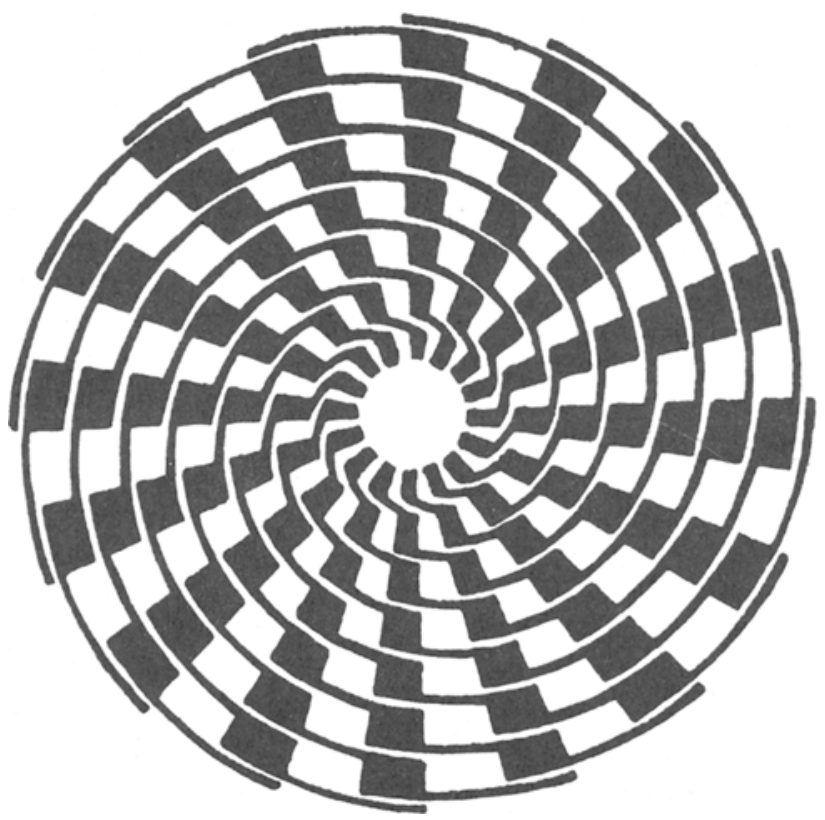

Fig. 2. Variation of the twisted cord illusion where the cord widths are constant as are the distances between the cords. The apparent depth is reduced. long enough to establish a strong iconic image which might be scanned internally (Sperling, 1960). Even if there is no internal scanning process, exposures shorter than $125 \mathrm{msec}$ theoretically may cause certain features of the display to be lost while retaining others (Rummelhart, 1970). Thus, short exposures may produce a change in the saliency of the different characteristics of the figure even though eye movements are eliminated.

(d) There are two integrative processes: one is the confluent union of units across the stippled areas in Fig. la, and the other involves the collective trends of the units of direction. Fraser further surmised that the first of these integrative processes is at work in the foveal region and that the second of these prevails at the periphery of vision. The neural architecture of the retina argues against the implication that different retinal processes are at work, since one would expect a "confluent union" of two adjacent dark areas to occur where the resolving power of the retina is weakest, viz, the periphery. However, the lines in the figure itself become finer in its central parts. Thus, "confluent union" may take place in the center of the figure as Fraser suggested, not so much because of the structure of the retina, but rather because of the characteristics of the physical stimulus.

A word is in order about Fraser's use of the term "confluence." Fraser adhered closely to the original meaning of the word proposed by Mueller-Lyer, who suggested that distances between closely spaced stimuli appear diminished (Hochberg, 1971). This is similar to the Gestalt notion of "closure." As for the term "collective trend," Fraser did not explain what this entailed. This term will appear later in this paper, at which time it will be given more explicit meaning.

Two different hypotheses concerning the illusion emerge upon reflecting on Fraser's four points. The first is that the twisted cord effect is influenced by the presence of simpler illusions embedded in it. This hypothesis will be called the illusion component 
Fig. 3. (a) Zöllner's illusion. (b) Fraser's version of the reversed Zöllner illusion. [The slashes are angled $11 \mathrm{deg}$ (top) and $15 \mathrm{deg}$ (bottom) from the line.]

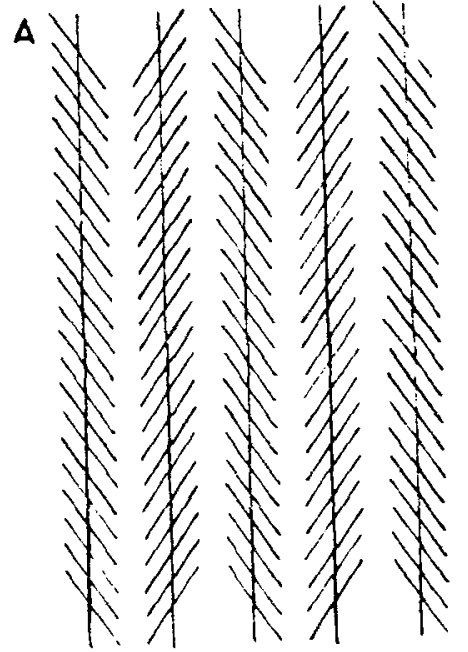

B
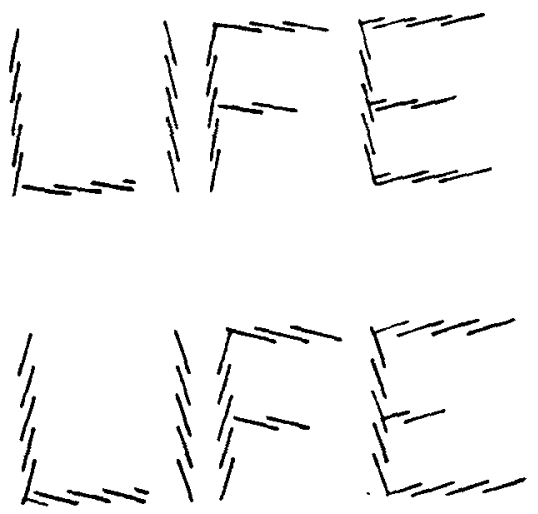

hypothesis. The other hypothesis, the one favored by Fraser, stresses the importance of the units of direction as independent components without reference to their connection with simpler illusions. The suggestion that these units are independent suggests such processes as eye movements or internal scanning. Although Fraser did not mention internal scanning, his use of words like "direction" and "trend" implies that he might have readily accepted such a construct had it been suggested to him. This hypothesis will be called the unit of direction hypothesis.

The remainder of this paper will be spent in discussing three variants of Fraser's original figure and their consequences for the several theoretical statements discussed above. There will be no guarantee that the issue regarding the two general hypotheses referred to above will be resolved. If there is to be a stated purpose to this paper, it is this: A number of variations of the twisted cord illusion are possible, and in the course of dealing with these variations, there may occur some interesting (and important) consequences for theory.

\section{FIRST VARIANT: DISTORTING THE BACKGROUND SPIRALS}

The points in Fraser's units of direction, in conjunction with the stippled area, form the basis of two antagonistic sets of background spirals that may possibly influence the effect of the illusion. These spirals are quite evident in Fig. 1a. In order that these secondary spirals be brought into clearer focus, the stippled areas were filled in (the confluent union was provided by E) and one set of spirals eliminated. The resulting construction is reproduced in Fig. $\mathrm{lb}$. Since the secondary spirals are the basis of the alternating black and white background stripes, they will be called the "stripe spirals." There is another set of spirals apparent in Fig. 1 b. These spirals will be called "stepped spirals" and are found by tracing any black (or white) area clockwise from the edge to the center. Another interesting (and aesthetic) feature of these constructions is that the stepped spirals have perfect figure-ground symmetry.

The effects of these background spirals were tested empirically by first distorting them so that they ran against the apparent spiral of the twisted cord, then asking Ss if the cord still appeared to spiral. These distortions are given in the constructions shown in Figs. $1 b-1 f$. The point of zero curvature for the stripe spirals is given in Fig. 1c, and the stepped spirals of zero curvature are produced in Fig. le. In Fig. 1b, both spiral forms twist in the same direction as the illusion spiral, and in Fig. If, both types of secondary spirals run counter to the illusion. The degree of spiralness of these background components is given in the figure legend. Their spiralness was measured by the number of degrees they crossed as they turned from the outer edge to the inner edge. Since it will be useful to speak of these spirals in terms of their direction, any spiral line that leads inwardly as it is traced clockwise will be called a clockwise spiral. Counterclockwise spirals are correspondingly defined.

\section{EXPERIMENT I}

\section{Method}

\section{Subjects}

Ten undergraduate students, both male and female, with at least $20 / 30$ corrected vision were used. Each $S$ was nun individually.

\section{Stimuli}

The five constructions shown in Figs. 1b-1f, their mirror images. and a single black eight-armed spiral were drawn on individual cards. They were approximately $15 \mathrm{~cm}$ in diam.

\section{Procedure}

Each $S$ was shown the spirals at a normal reading distance. The furst. the black eight-armed spiral, was used to explain what 


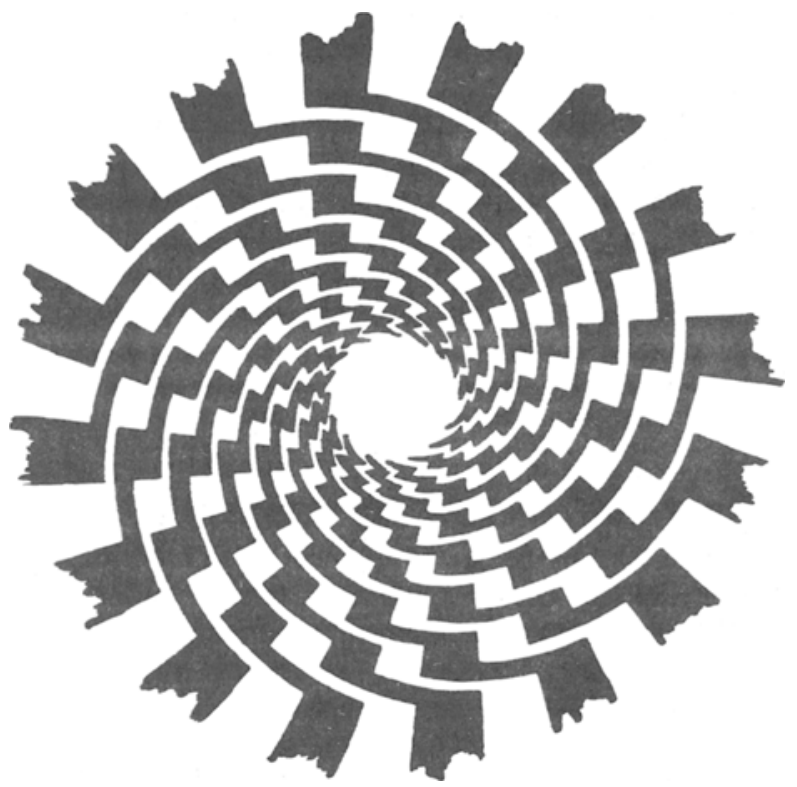

Fig. 4. Variation of the twisted cord illusion where the stripe spirals are linear and tangent to the inner edge.

was meant by "clockwise" and "counterclockwise" spirals. The $S$ was then told that some figures would be presented which showed a black and white stranded twisted cord superimposed on some background lines that radiated from the center and that he would be asked to make a judgment concerning the clockwise or counterclockwise direction of the twisted cord. The twisted cords in Figs. 1e and If tend to be hidden in the confusion of the background lines, and since the cords are difficult to see here, one of the other three figures (or its mirror image) was presented first. In spite of this precaution, some $S$ s found it difficult to see the cords in Figs. $1 \mathrm{e}$ and $1 \mathrm{f}$, so the following procedure was employed for all constructions. First, $E$ made sure $S$ saw the cord in Fig. 1b, 1c, or 1d. Then $E$ placed a pencil tip directly over the first cord ring in from the outside edge and traced it not more than halfway around the circumference. Then $E$ told $S$, "I am going to trace the cord around with my pencil tip, and I want you to tell me if the cord I'm following is spiraling clockwise or counterclockwise, or if my pencil has started to spiral inwardly or outwardly." The movements of the pencil were slow and proceeded in a clockwise direction for all Fig. 1 constructions and their mirror images. The five Fig. 1 constructions and their mirror images were presented to $S$ in random order.

\section{Results}

Of the $10 \mathrm{Ss}$, all but one produced responses consistent with the illusion in all the constructions shown in Fig. 1 and their mirror images. The probability of these results occurring by chance according to a binomial test is .011 . The one aberrant $S$ produced responses that can best be described as random. All of the figures were presented to her once again, and she was asked whether she saw circles or spirals in each. She reported circles in every case. The reasons for this unusual behavior are not known, and an attempt to explain it will not be pursued here.

\section{Discussion}

\section{Confluent Union}

Fraser suggested that at small visual angles, a confluent union would occur across the points between the units of direction located in successive rings. This confluence, supplied by $\mathrm{E}$ in Figs. 1b-1f, created both the stepped spirals and the stripe spirals seen in the constructions. It is evident that the direction of the stripe spirals was not solely responsible for the illusion's effect, since these spirals turn against the twisted cord in Figs. 1b-1d and the illusion was not destroyed. The same can be said of the stepped spirals; they become progressively steeper from Fig. $1 \mathrm{~b}$ to Fig. 1d, and the illusion did not vary extensively. More critically, the illusion was still intact in Figs. 1e and 1f, once the cord was teased out perceptually. Here the turn of the stepped spirals reaches zero and even runs counter to the illusion.

\section{Illusion Component Hypothesis}

The possibility of the twisted cord effect being determined by simpler illusion components was discussed earlier with particular reference given to the lay of the strands relative to the cord line. Figure 1c suggests another simple illusion effect created by the lay of the strands across the radial lines. Since the strands cross the radial lines at larger angles (closer to $90 \mathrm{deg}$ ), the situation here is conducive to Zöllner's illusion (see Fig. 3a). If the slashing lines produce an apparent opposite tilting of the radial lines, then the radials would appear off-center-perhaps tangent to some smaller circle drawn around the center point. Imagine that the center point strongly fixes the direction of the radial lines, thus creating a conflict between this fixation and the tilt produced by the Zöllner effect. Might this be resolved by an apparent spiraling of the cord? Figures $1 \mathrm{~b}-1 \mathrm{e}$ argue against this suggestion, since the radial lines in these constructions were varied past the point of the perpendicular, thereby eliminating the Zöllner effect, and yet the twisted cord illusion persisted. Furthermore, a construction was made in which the radial lines were linear, somewhat perpendicular to the units of direction, and tangent to the inner edge. The illusion was still intact in this construction (see Fig. 4).

\section{Conclusions}

The variations in Fig. 1 suggest that a Zöllner-type illusion imposed on the radial lines of the twisted cord figure either does not exist or, if it does exist, has little or no effect on the twisted cord illusion. The process of confluent union as manifested in either the stripe or stepped spirals also plays a minor part, if any, in the perception of the illusion. There remains the effect of the units themselves. These may produce the illusion 
Fig. 5. (a) A reconstruction of Fig. 1c with the radial lines perpendicular to the cord lines. (The stepped lines are spiral; the cord lines are circular.) (b) Half exchange of the circle and spiral lines of Fig. 1c. (Both the stepped lines and cord lines are spiraied.) (c) Full exchange of the circles and spiral lines of Fig. 1c. (The stepped lines are circular and the cord lines are spiraled.)

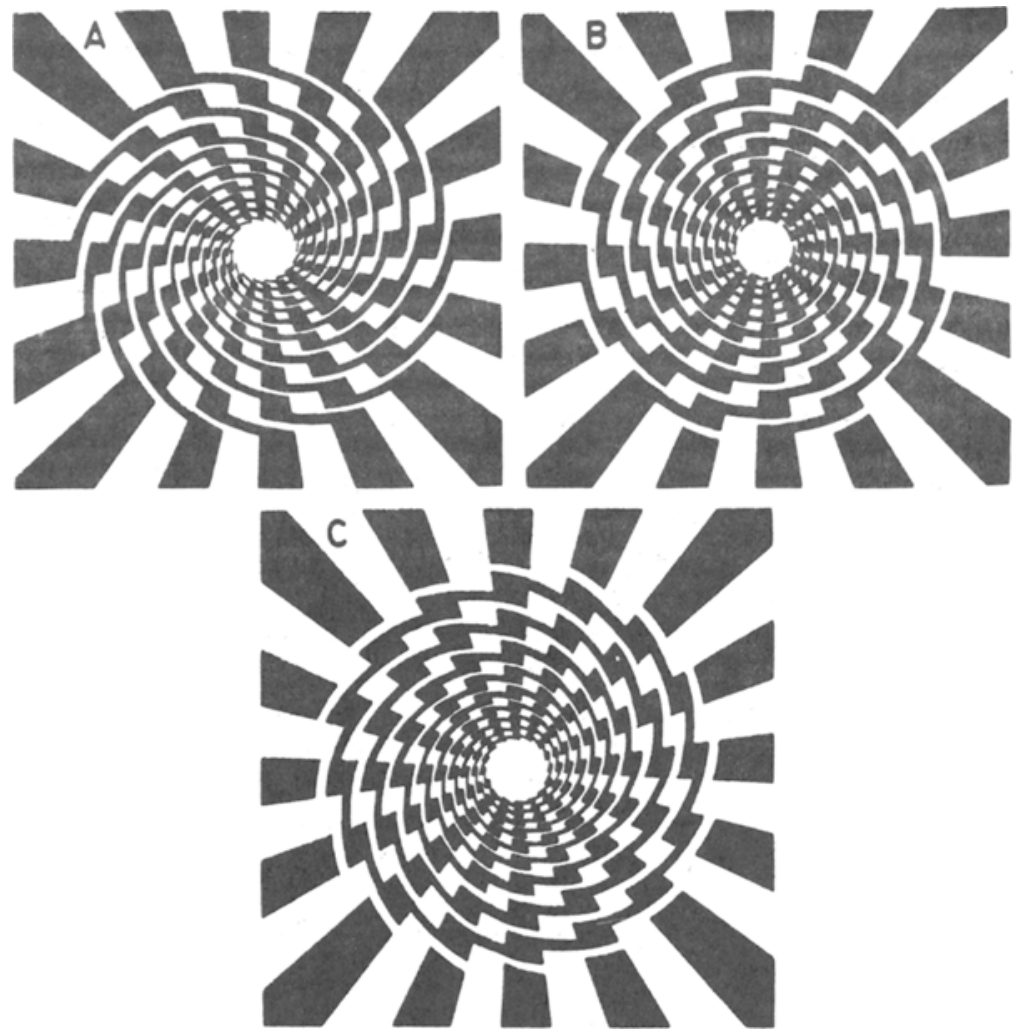

either by their direction ("collective trends," as Fraser put it) or by the simpler illusion component produced by these units and their relationship with the cord rings (the reversed Zöllner illusion). These constructions provided no test for either of these hypotheses.

\section{SECOND VARIANT: CIRCLE AND SPIRAL EXCHANGE}

In Fig. 5a (which is essentially the same as Fig. 1c), the stepped line is spiraled in a clockwise direction and the twisted cord describes a circular line. Thus, there is at least one circle line and one spiral line. (The terms "circle line" and "spiral line" should not be taken literally, i.e., indicating a continuous and completed line. Rather, these terms are used here to describe the suggestions of circles or spirals given by line segments or sequences of arcs.) In Fig. 5c, the situation is reversed; the twisted cord is spiraled in a clockwise direction and the stepped line is circular. Again, there is at least one circle line and one spiral line. In Fig. 5b, the stepped line spirals counterclockwise and the twisted cord spirals clockwise. There are no circle lines in this figure in the sense that there are circle lines in Figs. 5a and 5c. However, in this construction (5b), the units of direction are congruent to the circle lines rather than cutting across them. In Figs. 5a and $5 \mathrm{c}$, the units cut across the circumferences and follow what might be regarded as a spiral path to the center. These characteristics of the three figures are summarized in the first three columns of Table 1. Note that each figure has one circular line and two spiral lines associated with it.

In an informal query, several colleagues were shown the three constructions in Fig. 5 and asked to picture the radiating stripes as appearing on the inside of a tube with the twisted cord placed against the sides like rifling in a gun barrel. When asked to judge the shape and direction of the cord in Fig. 5a, there was no disagreement that the cord appeared spiraled in a clockwise direction. Similarly, Fig. 5c was unequivocally judged to be spiraled clockwise. The judgment of Fig. $5 \mathrm{~b}$, on the other hand, was a different matter. The responses were typically ones of perplexity and confusion. Some said the cord appeared circular; others said it spiraled, and when asked to trace it, they invariably selected the stepped spiral and traced it counterclockwise. When told that they were not tracing the twisted cord, their behavior degenerated into absolute frustration. Those who reported circles in Fig. $5 b$, when asked to trace a circle, also became agitated upon finding no circle to trace, just as they were perplexed when they could find no spirals in the cord lines of Fig. Sa. When asked to compare Fig. 5a and Fig. 5b, all selected Fig. 5b as being the more circular of the two.

When Figs. $5 b$ and $5 c$ were reduced in size to less than $5 \mathrm{deg}$ of visual angle, another characteristic not present in the Fig. 1 constructions became evident; perception at this visual angle appeared to be determined by the stepped spiral. That is, Fig. 5c appeared to be a set of nested circles, and Fig. $5 b$ looked spiraled in a 
Table 1

Summary of the Line Characteristics for Figures $5 a, b$, and $c$ and the General Results of Experiment 11

\begin{tabular}{|c|c|c|c|c|c|c|c|}
\hline & \multicolumn{3}{|c|}{ Direction of Line Relative to Center } & \multicolumn{2}{|c|}{$\begin{array}{l}\text { Bend of Units } \\
\text { Relative to: }\end{array}$} & \multicolumn{2}{|c|}{$\begin{array}{l}\text { Experiment II } \\
\text { Perception at: }\end{array}$} \\
\hline & $\begin{array}{l}\text { Cord } \\
\text { Lines }\end{array}$ & $\begin{array}{l}\text { Stepped } \\
\text { Lines }\end{array}$ & $\begin{array}{c}\text { Unit } \\
\text { Lines }\end{array}$ & $\begin{array}{c}\text { Radials } \\
\text { (Zollner) }\end{array}$ & $\begin{array}{c}\text { Cords } \\
\text { (Reversed } \\
\text { Zollner) }\end{array}$ & Limen*- & $\begin{array}{l}\text { Supra- } \\
\text { limen }\end{array}$ \\
\hline $\begin{array}{l}\text { Fig. } 5 \mathrm{a} \\
\text { Fig. 5b } \\
\text { Fig. 5c }\end{array}$ & $\begin{array}{l}\text { Circles } \\
\text { Spirals } \\
\text { Spirals }\end{array}$ & $\begin{array}{l}\text { Spirals } \\
\text { Spirals } \\
\text { Circles }\end{array}$ & $\begin{array}{l}\text { Spirals } \\
\text { Circles } \\
\text { Spirals }\end{array}$ & $\begin{array}{l}\text { Inward } \\
\text { Perpendicular } \\
\text { Inward }\end{array}$ & $\begin{array}{l}\text { Inward } \\
\text { Outward } \\
\text { Outward }\end{array}$ & $\begin{array}{l}S_{p} \rightarrow C_{I} \\
C_{I} \rightarrow C_{r} \\
S p \rightarrow C_{I}\end{array}$ & $\begin{array}{l}\text { Spirals } \\
\text { Circles } \\
\text { Spirals }\end{array}$ \\
\hline
\end{tabular}

-The term $\mathrm{Cr} \rightarrow \mathrm{Cr}$ indicates the presence of a questionable limen. See text for numerical results.

counterclockwise direction. The predominance of the stepped line also manifested itself in the afterimages of the figures. Fraser reported that the illusion was present in the afterimage of his figure, and indeed the afterimage of Fig. 5a was strongly spiraled, However, the afterimage of Fig. $5 b$ was also spiraled, but in a counterclockwise direction. The afterimage of Fig. $5 \mathrm{c}$ was clearly circular. In spite of the evidence from Fig. 1 to the contrary, it looked as if Fraser's hypothesis concerning confluence, and the role the foveal area plays in its determination, had been temporarily revived. Stimulation of the more central portions of the retina seemed at least to force a change in the saliency of different features of the figure.

This change in saliency at different visual angles, and the ambiguity surrounding the judgment of Fig. 5b, warranted further investigation. It was felt that certain features of the display would be lost, or at least their saliency would change, under presentations of short duration and that this might prove to be informative. For example, suppose Fig. 5c appeared spiraled above some duration threshold and circular below it. This might suggest that the twisted cord is most salient at long durations and the stepped line is most salient at shorter ones. It would follow that both Fig. 5a and Fig. 5b, when presented at durations below the threshold of Fig. 5c, should look spiraled, since the stepped line is spiraled in both of these constructions. Furthermore, if the twisted cord is predominant at durations above the threshold found for Fig. 5c, Fig. 5b should at no time appear circular, since the cord line is also spiraled in this figure.

\section{EXPERIMENT II}

\section{Method}

\section{Subjects}

Twelve male and female undergraduate students with $20 / 30$ corrected vision and no evidence of lateral phoria were used. Each $\mathrm{S}$ was run separately.

\section{Apparatus and Stimuli}

A two-channel tachistoscope was used. The "home" channel contained a display of black and white stripes radiating from the center of the field. These stripes were identical to those in Figs. $5 a, b$, and $c$, except the twisted cord was missing. Each of the three constructions was positioned so that, during exposure, the radiating stripes exactly matched those seen in the home channel. The perceptual effect here was one of the continual presence of the radiating stripes with the twisted cord figure briefly superimposed on them during exposure. The figures subtended a visual angle well above $5 \mathrm{deg}$.

\section{Procedure}

A multiple staircase procedure was used with $\mathrm{S}$ judging all three figures. The presentations were limited to the following durations (in milliseconds): $1,000,500,250,100,90,80,70$, $60,50,40,30$, and 20 . Since each figure was started at both ends of this range, six staircase functions were plotted for each $S$, i.e., one ascending and one descending for each of the three constructions. The six staircase conditions were given in blocks of six trials, but they were random within that block. Each S was asked to report either circles or spirals. Their instructions read as follows: "If you look into the eyepiece you will see a set of lines converging on a point in the center. This is almost like looking down a tube with black and white stripes painted on the inside. I will superimpose one of three sets of lines on the ones you see here. Sometimes these will look like nested circles, sometimes like spirals. It will be as if there was rifling in the tube or a set of rings placed at equal distances down the tube. Even the same set of lines may look like circles or spirals on different occasions. I want you to tell me whether you see circles or spirals. Some of these will be shown very quickly, and if you find it difficult to give an answer, give me your general impression of what you saw. Also, since the presentations will be brief, I will give you a warning signal just before the image is flashed." Half the Ss were given longer durations on the next presentation if they reported seeing circles and shorter durations if they saw spirals. This was reversed for the other half of the Ss. If the ascending and descending staircase functions for the same figure diverged from the upper and lower limits during the first trials, the response criteria were reversed so that the two functions could converge. If the ascending and descending functions converged at the range limit, that duration was repeated for five trials and dropped if no reversal of the functions was found.

\section{Results and Discussion}

The point at which the two functions for a given figure first met was taken as the threshold for that figure. For example, for one $\mathrm{S}$, Fig. $5 \mathrm{c}$ was seen as spiraled at 1,000 msec and circular at $20 \mathrm{msec}$. The two functions met at $35 \mathrm{msec}$, and at this exposure duration, $S$ oscillated between a report of spirals and circles. In addition to the summary of the various characteristics of, and relationships between, the cords, units, and stepped spirals, Table 1 includes the results of Experiment II in nonquantitative terms. 


\section{Unit of Direction Hypothesis}

Of first importance is the fact that there was complete agreement among the Ss regarding the suprathreshold perceptions. Comparing Ss' suprathreshold responses with the other columns in Table 1, it can be seen that there was perfect agreement between Ss' perceptions of these figures and the lay of the units relative to the figures' centers. The Ss' suprathreshold perceptions of the three figures showed no such agreement with the lay of the cord or stepped lines. If Fraser's "unit trend" description can be interpreted in terms of the lay of the units relative to the center, then the suprathreshold responses reinforce the importance of these trends.

Column 6 in. Table 1 gives the Ss' change in perception at threshold. At some point above a $20-\mathrm{msec}$ exposure duration, $58 \%$ of the $\mathrm{Ss}$ changed their perception of Fig. 5a from spirals to circles and, in the case of Fig. 5c, 58\% switched their reports from spirals to circles. However, only $17 \%$ of the Ss saw Fig. 5b change from circles to spirals at durations greater than $20 \mathrm{msec}$. These are significant variations according to Cochran's $Q$ test $\left(\chi^{2}(2)=6.25\right)$.

These data tell us that the constructions in Figs. 5a and $5 \mathrm{c}$ have thresholds at which certain features become more salient, whereas the existence of such a threshold for Fig. $5 b$ is perhaps questionable. What is important for our purposes here is that the perception does not change consistently from stepped line to cord line, nor does it change from cord line to stepped line across all figures. Therefore, it is unlikely that the stepped line (or the cord line) plays a similar role across all three figures.

One thing the threshold data do not tell us is exactly what is happening to perception at threshold. A hint comes from the results of the informal survey mentioned earlier, that the perception of Fig. 5b was not all that clear. One possibility is that the subjective contours of the cord are lost or, at best, difficult to discern in Fig. 5b. These contours are easier to see in Fig. 5a (and $5 c)$; indeed, it is precisely these contours that give rise to the perception of the cord (but not their direction). Perhaps what is being measured in Experiment II is the presence and absence of subjective contours. This might help explain the fact that little evidence was found for a threshold in Fig. 5b, since there are no subjective contours there to be lost.

We are further cautioned by the fact that the stepped line (confluence between units) very obviously determines the perception of these figures at small visual angles. The arguments above apply only to larger projections of these figures on the retina, and it is apparent that two very different and independent processes are at work at large and small visual angles.

\section{Illusion Component Hypothesis}

In Figs. 5a and 5c, the units or strands of the cord tracing them clow wise. are slanted inwardly relative to the radial lines, but they are perpendicular to the radials in Fig. 5b. This was also correlated with Ss' perceptions. Recall that the relationship of the units to the radial lines tends to create a Zöllner effect. At the same time, the lay of the units relative to the radials defines the lay of these units relative to the center, and this fits the definition of "collective trend" used here (see Columns 3 and 4 of Table 1). Since Fig. 1 and Fig. 2 provided evidence against the influence of the Zöllner effect here, the importance of the Zöllner effect as an explanatory principle will be rejected in deference to the unit trends.

In the reversed Zöllner illusion, the line of the inducing slashes leans in the direction of the slashes (see Fig. 3b). In Fig. 5a, the strands of the cord, tracing them clockwise, slant inwardly relative to the cord lines. Thus, an apparent inward bending of the line should be produced, and since the line is circular, this apparent bending perhaps produces the spiral effect. But what about Figs. $5 \mathrm{~b}$ and $5 \mathrm{c}$ ? Here the units of direction point out wardly (again tracing them clockwise) from the spiral lines of the cord, thereby bending the spirals outward. perhaps into circles. If the reversed Zöllner illusion is responsible for this effect, would not the cord lines in both Figs. $5 \mathrm{c}$ and $5 \mathrm{~b}$ appear circular? Perhaps not. The cord lines in Fig. 5c spiral more steeply (lead to the center at a faster rate) than those in Fig. 5b. The cord lines in Fig. 5c may be too steep, whereas the shallowness of the cord lines in Fig. 5b may be precisely right for the illusion to counteract it and produce the apparent circles-an effect which, if true, would be surprisingly coincidental.

\section{Conclusions}

Again some question has been raised regarding the role of confluence as seen in the stepped spirals in producing the perception of this illusion. Yet the variations in Figs. 5a, b, and c suggest that the stepped lines control perception under conditions of small visual angle (foveal projection) and afterimages. On the other hand, the trends of the units of direction were consistent with the perception of larger representations of these figures. implying that this form of the unit of direction hypothesis is still viable. Furthermore, these figures point to an interesting interpretation of the term "unit trends." viz, that the trends of the units define still another set of spiral lines in the twisted cord figure.

Figures $5 \mathrm{~b}$ and $5 \mathrm{c}$, while raising some questions about the efficacy of the reversed Zöllner illusion, did not provide a crucial test of it. Thus, we are again left with two possible critical characteristics of the twisted cord figure the reversed Zöllner illusion component and the trends of the units of direction.

THIRD VARIANT: SEPAR ITION OF ALL UNITS OF DIRECTION

One way in which the effects of the units by then eloes might be examined would be to remove the 

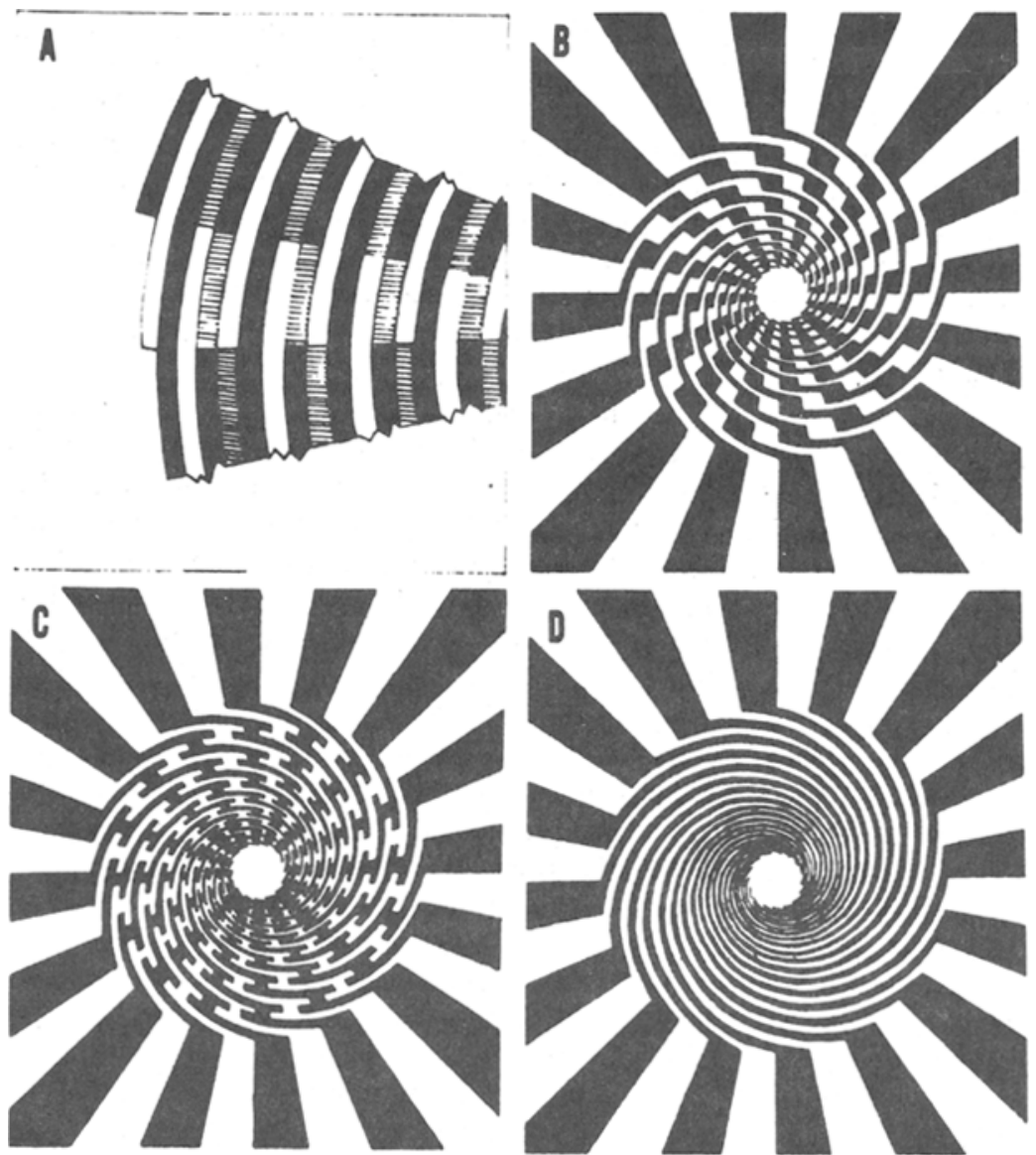

Fig. 6. The demonstration that the illusion spiral is the same as the units of direction spiral lines. (a) A section of the twisted cord figure with only the black and white units of direction. The lined areas were transparent. (b) A completed version of the $6 \mathrm{a}$ transparency superimposed on the radial stripes producing the twisted cord effect. (c and d) Clockwise rotation of the transparency in Fig. 6b through the distance of a single stripe width. background lines (the stripe spirals) completely, leaving only the individual units displayed. The resulting figure spirals weakly, which poses some problems since it has been suggested throughout this paper that the background spirals do not have a profound effect on the perception of the illusion. The observation made earlier that the constructions in Fig. 1 have perfect figure-ground symmetry provides some help here. Because figure-ground symmetry exists, there are white as well as black units of direction, and the removal of the black stripe spirals effectively eliminates these white units of direction. Therefore, in order to study appropriately the effects of the units of direction, both white and black units must be kept intact.

Figure 6 demonstrates the way in which these units and their trends were studied by parceling the black and white units intact and then gradually phasing out the background lines. The type of spiral shown in Fig. 1c was used because the linear background lines simplified the construction. The black and white units of direction were painted on a transparency, leaving clear places represented by the lined areas in Fig. 6a. This transparency was laid over a set of black and white radial stripes whose widths matched the widths of the overlapping segments of the units. The rotation of the transparency through the width of a single radiating stripe produced a continuous change in pattern going from Fig. $6 \mathrm{~b}$ through $6 \mathrm{c}$ to $6 \mathrm{~d}$. The effect was one of doubling the number of spirals seen, but the degree of spiral turn seemed to remain constant.

This suggested a rather unusual interpretation of the twisted cord effect. First, Fraser's trends of the units may indeed be critical, since the slant of the units seem to lie along spiral lines. More importantly, these spiral lines may be the very ones seen in the illusion. We tend to say that the twisted cord in Fig. $6 \mathrm{~b}$ appears spiraled, but that the spirals really do not exist. Thus, we call the spirals "illusions." In point of fact, the spirals are real and, given the presence of subjective contours, the cord is real. The illusion is seen as the real cords lying along real spiral lines instead of lying along nonexistent spiral lines or their more veridical circular lines.

The following experiment was conducted to see if any substantial difference in the degree of spiralness existed between the spirals in Figs. 6b and 6d. One of the difficulties encountered here involved the possible change in the spiralness of Fig. $6 \mathrm{~d}$ due to the increased number of spirals rather than to the steepness of the spiral arms. Therefore, Fig. $6 \mathrm{~d}$ was not used in this experiment.

\section{EXPERIMENT III}

\section{Method}

Subjects

Ten male and female undergraduate students having at least 
Fig. 7. (a) Control figure to replace Fig. 6d. (b) 405-deg spiral. (c) 450-deg spiral. [The differences between (b) and (c) are perceptually greater than the judged differences between Figs. $6 \mathrm{~b}$ and 7a.]
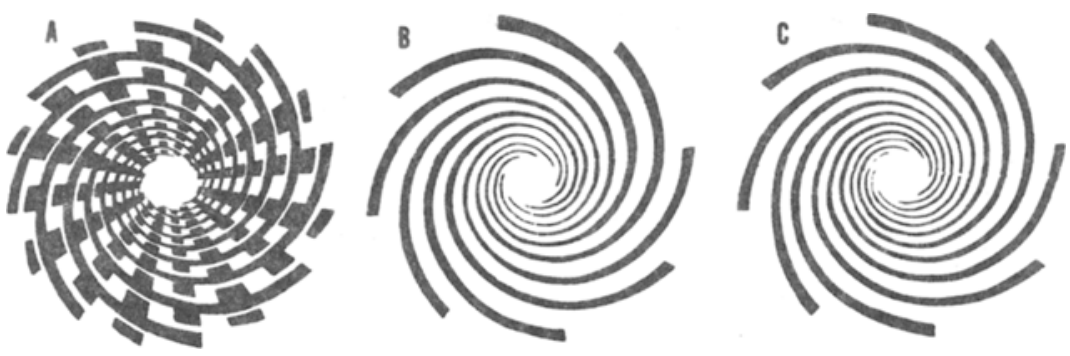

$20 / 30$ corrected vision were run individually.

\section{Stimuli}

The construction in Fig. 6b (without the extended radial stripes) was used as the illusion spiral, and the eight-armed spiral shown in Fig. 7a was used as a control spiral. The degree of spiralness of Fig. 7a was exactly the same as that of Fig. 6d. Nine eight-armed black spirals on a white background served as comparison spirals. The two extremes of the nine eight-armed spirals turned through 225 and $585 \mathrm{deg}$; the remaining seven were equally spaced between these extremes following increments of $45 \mathrm{deg}$. The mirror images of these spirals were also used. All spirals were about $15 \mathrm{~cm}$ in diam and were viewed at normal reading distance.

\section{Procedure}

The 225-deg spiral was called "steep" in this experiment, and the 585-deg spiral was referred to as "shallow." After assurances that $S$ knew what was meant by steep and shallow spirals, he was given the following instructions. "You will be shown pairs of spirals like this [S was shown the 225-deg comparison spiral with either the spiral shown in Fig. $6 \mathrm{~b}$ or Fig. 7a]. I want you to tell me if this spiral [E pointed to the comparison] is steeper or shallower than the other one. Don't be too analy tical in making your judgment. Just give me your impression of the relative steepness and shallowness of the two figures." The $E$ then paired the 225- and 585-deg comparison spirals with the two standard spirals (making four comparisons) and asked for S's judgments to make sure $S$ understood the task. A random multiple staircase procedure was used, and $S$ made judgments of both standard spirals. The standard and comparison spirals were shown side by side, but their positions were changed randomly from trial to trial. Clockwise and counterclockwise forms of the stimuli were shown on alternating trials in an attempt to hinder the satiation of the illusion.

\section{Results and Discussion}

The staircase functions of one $S$ showed no sign of merging after 10 trials, and her data were discarded. After the staircase functions given by the other Ss met, they were continued for six trials to check stability. If the two functions remained together and started to drift, the trials continued until they reached what seemed to be an asymptotic level. The means of the staircase values after the point of first contact were taken as the PSE.

The mean degree of spiralness the Ss selected as matching Fig. $6 \mathrm{~b}$ was $454 \mathrm{deg}$. The degree of spiralness equated with Fig. 7 a was $435 \mathrm{deg}$. The discrepancy of $19 \mathrm{deg}$ was not significant $[\mathrm{t}(8)=1.046]$. Even if it were, however, a judgment of the illusion spiral being shifted slightly toward shallowness would not be unexpected, since the possibility of satiating on the illusion was still present in spite of the precautions taken to eliminate this problem.

The purpose of this experiment was to show that the discrepancy between the perceptions of the spirals in Figs. $6 \mathrm{~b}$ and $7 \mathrm{a}$ was not outlandish. The two comparison spirals (405 and $450 \mathrm{deg}$ ) which most closely matched the mean judged spirals ( 435 and $454 \mathrm{deg}$ ) are given in Figs. $7 \mathrm{~b}$ and $7 \mathrm{c}$. Their differences are hardly excessive.

A final task remains, and this is to show which of the two factors, the reversed Zöllner or the direction of the units, is most responsible for the twisted cord effect. Adam demonstrated that the reversed Zöllner illusion in one of its linear forms weakened as the angle of the inducing slashes increased. Close comparison of Fig. 1c and Fig. $6 \mathrm{~b}$ reveals that the units of direction angles are slightly steeper in Fig. 6b than in Fig. 1c. In Fig. 6c, each unit is a continuation of a unit one step away on the next ring. In Fig. 1c, the continuation is two or three units away. In Fig. $6 \mathrm{~b}$, the units cross the cord line at about a 15-deg angle; in Fig. Ic, this angle is somewhat less (approximately 10 or $11 \mathrm{deg}$ ). The two constructions, therefore, might provide a critical test between the reversed Zölner illusion component hypothesis and Fraser's hypothesis of unit trend as interpreted here.

The unit of direction spiral is steeper in Fig. 6b than in Fig. 1c. If the unit of direction trends form the basis of spirals that govern the perception of the cord, then the cord in Fig. $6 \mathrm{~b}$ should be judged to be a steeper spiral than the cord spiral in Fig. 1c. On the other hand, the reversed Zöllner illusion component hypothesis would predict the contrary, since according to Adam's findings the more the units are angled away from the unit line, the less the line leans in the direction of the units. Thus, Fig. $6 \mathrm{~b}$ should appear more shallow than Fig. 1c. This pair of contradictory predictions prompted the following experiment.

\section{EXPERIMENT IV}

\section{Method}

\section{Subjects}

Twenty undergraduate students, male and female, with $20 / 30$ corrected vision were run. Each $\mathbf{S}$ viewed the display.s individually. 
Table 2

Frequency of Responses for Each Figure in Experiment IV

Figure and Judgment

\begin{tabular}{cccc}
$\begin{array}{c}\text { Angle of } \\
\text { Inducing } \\
\text { Lines }\end{array}$ & $\begin{array}{c}\text { LIFE } \\
\text { (More } \\
\text { Crooked) }\end{array}$ & $\begin{array}{c}\text { Twisted } \\
\text { Cord } \\
\text { (Steeper) }\end{array}$ & $\begin{array}{c}\text { Conven- } \\
\text { tional } \\
\text { Spiral } \\
\text { (Steeper) }\end{array}$ \\
\hline 11 Deg & 11 & 4 & 3 \\
15 Deg & 9 & 16 & 17 \\
\hline
\end{tabular}

\section{Stimuli}

Four pairs of displays were used. The critical pair included twisted cord constructions similar to those shown in Figs. 1c and $6 \mathrm{~b}$; the angles with which the units crossed the circular cord lines were 15 and $11 \mathrm{deg}$. Two units of direction control spirals were shown. These were two black spirals on a white background that matched the spiralness of the unit of direction spirals in Figs. 1c and $6 \mathrm{~b}$. Two reversed Zöllner illusion control figures were shown. These were the two LIFE displays shown in Fig. 3b. The angles of the inducing slashes in the top LIFE figure are $11 \mathrm{deg}$, and the slashes were made to tilt 15 deg away from the line in the lower figure. This, of course. matched the unit of direction angles in the two twisted cord constructions used in the experiment. Two black spirals on a white background which turned through 585 and 225 deg served as practice spirals.

\section{Procedure}

The Ss were told that they would be shown a pair of spirals and that they were to judge which spiral was steep and which was shallow. The $E$ then drew a steep and shallow spiral to make sure $S$ understood what was meant by these descriptions. The $S$ was then shown the practice spirals to further assure $E$ that he knew what was meant by steep and shallow spirals. The $S$ judged the unit of direction control spirals, then the twisted cord illusion spirals. Following this, $E$ showed $S$ the two LIFE figures and asked $S$ to tell him which of the two LIFE figures contained letters which were the most crooked. The position of each display within each pair varied between Ss.

\section{Results and Discussion}

The results appear in frequency form in Table 2. Only 3 of the $20 \mathrm{Ss}$ misjudged the unit of direction control spirals. The 15-deg unit of direction control spiral was clearly seen as the steeper of the two. The Ss were almost evenly split in their judgments of the illusion component control figures, although there was a slight tendency to judge the 11-deg LIFE figure as more crooked than the 15-deg figure. This is somewhat in accord with Adam's findings. Thus, according to the illusion component hypothesis, the Ss should be at least evenly divided in their judgments of the two twisted cord figures, if not slightly favoring the 11-deg spiral as steeper. The unit trend hypothesis suggests that the Ss should favor the 15-deg unit of direction illusion (Fig. $6 \mathrm{~b}$ ) as being steeper. As shown in Table 2, the unit trend hypothesis received more support from these data than did the illusion component hypothesis. According to a McNemar test for significance of changes, the differences between the judgments of the LIFE and twisted cord figures were significant $\left[\chi^{2}(1)=5.1\right]$, whereas the judgment differences between the twisted cord and conventional spirals were not $\left[\chi^{2}(1)<1\right]$.

An objection might be raised here concerning the differences between the two figures as seen in the widths and distances between the cord lines. The greater distances between the cord lines in Fig. 1a increase the effect of depth in this figure. While depth cues cannot be held responsible for the twisted cord effect (see Fig. 2), they might enhance it slightly, and this would work against the unit trend hypothesis in this experiment. The widths in Fig. Ic should make the cord in this figure appear more spiraled rather than less, which is possibly why the number of Ss judging Fig. 1c as "steeper" was $4 / 20$ rather than $3 / 20$.

\section{THEORIES OF ILLUSIONS}

To say that the units of direction account for most of the illusion's effect only pushes the question back one step further. We have merely isolated the responsible component, but we have not described how this component produces the illusion. The best we can do in answering this question is to look at some theories of illusions and how they relate to the twisted cord effect.

\section{Confusion Theory}

According to confusion theory, components extraneous to a judged component can be seen as part of or an extension of that judged component. Thus, the fins of the Mueller-Lyer illusion create a total impression of added length, and the observer cannot eliminate this impression from his judgment. There are any number of spiral components in the twisted cord figure which could influence the judged direction of the cord line. The experiments described herein point to the spiral produced by the units of direction as being dominant in this respect.

Given that the cord strand spirals are prepotent, is it possible that they might interact with the perception of the cord in a way suggested by confusion theory? Experiments III and IV demonstrated that the spiralness of the circular cord matched the strand spiral lines. Thus, there is evidence that the judged cues are influenced by impressions created by extraneous cue lines, and this satisfies the conditions of confusion theory.

Confusion theory is more of a descriptive theory than a process theory, however. It describes what the effects of the added components are, but it does not describe how these effects are produced.

\section{Lateral Inhibition}

Ganz (1966) has developed a lateral inhibition theory for figural aftereffects. This is a process theory which presumably could apply to figures such as the twisted 
cord illusion. The optical aberration theory of Chiang (1968) would also belong in this class. In its simplest terms, Ganz's theory states that lateral inhibition is responsible for apparent contour displacements when those contours are separated by small distances. Furthermore, this inhibition diminishes as the distance between the contours decreases. The intersections of the striped lines and the units of direction are the most likely loci for the lateral inhibition effect in the twisted cord figure. Note that the points of intersection vary in angle size from Fig. $1 \mathrm{~b}$ to $1 \mathrm{f}$. One would expect a systematic change in the illusion across these figures, since the change in angle represents variations in the distance between the critical contours. Perhaps the biggest problem for the theory of lateral inhibition, though, is that it does not explain why the apparent spiral of the cord coincides with the spiral of the units of direction as suggested by Experiments III and IV.

\section{Eye-Movement Theory}

Since there are numerous spiral components in the twisted cord figure, it would seem natural to assume that these components would be followed by the eye, and this in turn would give rise to the apparent spiraling of the cord. This is particularly appealing since the eye might trace the unit of direction spiral line, thereby explaining the results of Experiments III and IV. Yet the illusion persisted at durations shorter than $100 \mathrm{msec}$, which indicates that eye movements are not necessary for this effect.

However, the results of Festinger, White. and Allyn (1968) suggest that it is the efferent commands that are responsible for the perception of some illusions and not the eye movements themselves. They noted that saccadic movements across two lines of equal length were different for each line when the two lines were embedded in an illusion such as the Mueller-Lyer figure. As time passed, the saccadic movements equalized and the illusion was lost. Yet the illusion did not disappear, nor did it fade when their Ss fixated at a point central to the figure. In both instances, efferent commands were made. In the first instance, these commands were being "recalibrated" by the saccadic movements, as Festinger et al put it, and the illusion was lost. In the second instance, there was no "recalibration" and the illusion remained intact.

Scanning the twisted cord is quite different from scanning the Mueller-Lyer illusion, where the saccadic movements are repeated over the same line. While the efferent commands accompanying the perception of the twisted cord may always be commands to trace spirals equal to the unit of direction lines, they probably take different directions. As the figure is scanned, the eyes are likely to move from the outer to the inner edge. They may also tend to skip clockwise, sampling different spiral arms as they go. Thus, the efferent command has a fixed component (outer to inner edge) and a variable one resulting from the clockwise skipping (left to right, up to down. right to left, down to up. etc.). If there is a component of the command that changes continuously, there may be resistance to command "recalibration," and this is why this illusion does not satiate as readily as some of the simpler illusions.

Figure 5b still presents a problem. One sees circles in this figure which may well be produced by an efferent command to follow circular units of direction. However, the perception of this figure is probably one of general circularity and not one of concentric rings of twisted cords. It was suggested earlier that this is perhaps due to a loss of subjective contrast in this figure. But the question remains whether or not the loss of subjective contrast is in any way related to the change in efferent commands from spirals to circles. It is quite possible that subjective contrast is related to the circularity of the cord and not to the direction of the units. If so, then subjective contrast would be present only in Fig. 5a and missing in Figs. $5 b$ and $5 c$. Note that this contradicts the assumptions concerning the relationship between subjective contours and perception at threshold made in the discussion of Experiment II.

\section{A Multicomponent Theory}

With a figure as complex as the twisted cord illusion, it might seem likely that there are numerous factors which contribute to the effect. Taking Fig. $1 \mathrm{c}$ as an example, the Zöllner illusion imposed on the radial lines. the reversed Zöllner illusion seen in the circular lines, the spiralness of the units of direction, the spiralness of the stepped lines, and the apparent depth created by the width of the cord lines and the distances between them all might contribute in some small but additive way to the apparent spiralness of the cord. The experiments described here required, for the most part, one bit decisions (clockwise-counterclackwise, circles-spirals, etc.); elimination of cues only established the necessity (or lack of it) of that cue for the creation of the illusion. Hence, we can say that the stripe spiral was not a necessary cue, since it was eliminated in Fig. 1c and the illusion remained. The question of the degree to which the stripe spirals contributed to the illusion in the remaining constructions in Fig. 1 was not answered here. One would have to extract judgments concerning the magnitude of the effect across all constructions in Fig. 1 in order to answer this. The isolation of the change in effect produced by changes in a single cue may be formidable, since often one cue cannot be varied independently of another, as in the case of the stripe and stepped spirals.

\section{REFERENCES}

Adam, J. A note on visual illusions of direction. Australian Journal of Psychology. 1964. 16. 53-56. 
Chiang, C. A new theory to explain geometric illusions produced by crossing lines. Perception \& Psychophysics, 1968, 3, 174-176.

Dufour, M. Illusion d'optique relative an micrometreocculaire de Krauss. Comptes Rendus Hebdomadaires de Seances de l'Academie de Sciences (Sociéte de Biologie), 1930, 105, 600-601.

Festinger. L., Ono, H., Burnham, C., \& Bamber, D. Efference and the conscious experience of perception. Journal of Experimental Psychology Monograph, 1967, 74(4, Whole No. 637).

Festinger, L., White, C. W., \& Allyn, M. R. Eye movements and decrement in the Müller-Lyer illusion. Perception \& Psychophysics, 1968, 3, 376-382.

Fraser, J. A new visual illusion of direction. British Journal of Psychology, 1908, 2, 307-320.

Ganz, L. Mechanisms of the figural aftereffects. Psychological Review, 1966, 73, 128-150.

Hochberg, J. Perception 1: Color and shape. In J. W. King and L. Riggs (Eds.), Woodworth and .. Schlossberg's experimental psychology'. (3rd ed.) New York: Holt, Rinehart \& Winston, 1971. Pp. 395-474.

Hyde, W. F. A variant of the chessboard illusion. American
Journal of Psychology, 1929, 41, 296-297.

Imai, S. Experiments on negative illusions in Zölner's Figure. Jimbun Gakuho (Journal of Social Sciences \& Humanities), Toyko Metropolitan University, 1962, No. 30, 31-44.

Parola, R. Optical art: Theory and practice. New York: Van Nostrand Reinhold, 1969.

Rummelhart, D. E. A multicomponent theory of the perception of briefly exposed visual displays. Journal of Mathematical Psychology, 1970, 7, 191.216.

Sperling, G. The information available in brief visual presentation. Psychological Monographs, 1960, 74 (No. 11).

\section{NOTE}

1. A variation of the twisted cord illusion drawn by Betty Porter, a student of Rene Parola, was pointed out to me which bears a striking resemblance to the construction in Fig. 2 (see Parola, 1969, p. 50).

(Received for publication January 22, 1973; revision received July $9,1973$. ) 